\title{
Novel EUV mask absorber evaluation in support of next-generation EUV imaging
}

Vicky Philipsen, Kim Vu Luong, Karl Opsomer, Christophe Detavernier, Eric Hendrickx, et al.

Vicky Philipsen, Kim Vu Luong, Karl Opsomer, Christophe Detavernier, Eric Hendrickx, Andreas Erdmann, Peter Evanschitzky, Robbert W. E. van de Kruijs, Zahra Heidarnia-Fathabad, Frank Scholze, Christian Laubis, "Novel EUV mask absorber evaluation in support of next-generation EUV imaging," Proc. SPIE 10810, Photomask Technology 2018, 108100C (10 October 2018); doi: 10.1117/12.2501799

Event: SPIE Photomask Technology + Extreme Ultraviolet Lithography, 2018, Monterey, California, United States 


\title{
Novel EUV mask absorber evaluation in support of next-generation EUV imaging
}

\author{
Vicky Philipsen ${ }^{1, *}$, Kim Vu Luong ${ }^{1,2}$, Karl Opsomer ${ }^{1}$, Christophe Detavernier ${ }^{3}$, Eric Hendrickx ${ }^{1}$, \\ Andreas Erdmann ${ }^{4}$, Peter Evanschitzky ${ }^{4}$, Robbert W.E. van de Kruijs ${ }^{5}$, Zahra Heidarnia-Fathabad ${ }^{5}$, \\ Frank Scholze ${ }^{6}$, Christian Laubis ${ }^{6}$ \\ ${ }^{1}$ imec, Kapeldreef 75, B-3001 Leuven, Belgium \\ ${ }^{2}$ KU Leuven, Department of Materials Engineering, Belgium \\ ${ }^{3}$ University of Ghent, Cocoon, Department of Solid State Sciences, Belgium \\ ${ }^{4}$ Fraunhofer IISB, Schottkystr. 10, 91058 Erlangen, Germany \\ ${ }^{5}$ University of Twente, PO Box 217, 7500 AE Enschede, The Netherlands \\ ${ }^{6}$ PTB, EUV Radiometrie, Abbestr. 2-12, 10587 Berlin, Germany
}

\begin{abstract}
In next-generation EUV imaging for foundry N5 dimensions and beyond, inherent pitch- and orientation-dependent effects on wafer level will consume a significant part of the lithography budget using the current Ta-based mask. Mask absorber optimization can mitigate these so-called mask 3D effects. Thin metal absorbers like Ni and Co have been experimentally investigated due to their high EUV absorption, but they pose challenges on the current technology of subtractive mask patterning [1]. A simulation study of attenuated EUV phase shift masks has identified through multiobjective optimization superior imaging solutions for specific use cases and illumination conditions [2].

Evaluating novel EUV mask absorbers evolves on two levels, demonstrating (1) improvements from lithographic perspective and (2) compatibility with the full mask supply chain including material deposition, absorber patterning, scanner environment compatibility and mask lifetime.

On the lithographic level, we have identified regions based on the material optical properties and their gain in imaging performance compared to the reference Ta-based absorber. Within each improvement region we engineered mask absorber materials to achieve both the required imaging capabilities, as well as the technical requirements for an EUV mask absorber. We discuss the material development of Te-based alloys and Ag-based layered structures, because of their high EUV extinction. For the attenuated phase shift materials, we start from a Ru-base material, due to its low refractive index, and construct Ru-alloys.

On the experimental level, we examined our novel mask absorber materials against an initial mask absorber requirement list using an experimental test flow. Candidate materials are evaluated on film morphology and stability through thermal, hydrogen, EUV loading, and chemical cleaning, for their EUV optical constants by EUV reflectometry, as well as preliminary for selective dry etch.
\end{abstract}

The careful mask absorber evaluation, combining imaging simulations and experimental material tests, allowed us to narrow down to promising combinations for novel EUV mask absorbers.

Keywords: EUV mask absorber, mask 3D effects, absorber characterization, rigorous mask 3D lithography simulation

\section{INTRODUCTION}

Over the recent years the knowledge has grown and spread on mask-induced imaging effects, experimentally observed in EUV lithography for N5 dimensions and beyond, with the current EUV mask [1-9]. More precisely, the current Ta-based absorber is at its limit for imaging extendibility. Thinning down below 50nm Ta-based absorber thickness will reduce the amount of absorbed light, reduce the NILS and increase best focus variation through pitch $[10,11]$. Although the current Ta-based mask has proven benefits from mask technology point, the imaging performance towards next technology nodes can benefit from mask optimization. The industry needs to reconsider the EUV mask concept as a mitigation of mask-induced imaging effects (by balancing the diffraction for all features and pitches simultaneously).

Photomask Technology 2018, edited by Emily E. Gallagher, Jed H. Rankin, Proc. of SPIE Vol.

10810, 108100C · C 2018 SPIE · CCC code: 0277-786X/18/\$18 · doi: 10.1117/12.2501799 
Different concepts have been introduced over the recent years, from tuning the Mo/Si multilayer mirror periodicity [12], to changing the multilayer mirror materials to $\mathrm{Ru} / \mathrm{Si}$ [13] over embedding the absorber in the multilayer mirror [3] and the etched multilayer mirror mask $[14,15]$. Realizing these mask concepts in production worthy environment turned out difficult. Today, the focus of the industry is on novel mask absorber as the most realistic implementation towards future technology nodes. This became clear in the 2018 imec organized forums gathering the EUV blank supply chain to initiate a mask absorber change [16]. In this paper we report the careful evaluation, combining rigorous imaging simulations and experimental absorber material tests, of potential novel EUV mask absorbers.

In Section 2 we motivate the choice of alternative mask absorbers based on their imaging performance compared to the reference Ta-based mask for typical N5 building blocks at NA0.33. Section 3 details out the mask absorber requirements and we demonstrate our experimental evaluation methodology flow using our engineered, novel EUV mask absorbers. Section 4 summarizes the main findings of this paper and points to a path forward in the absorber material downselection.

\section{IMAGING GAIN IN EUV MASK ABSORBER CHANGE}

In this Section we highlight the benefit of a mask absorber change by projecting a wide range of imaging simulation results onto the material optical property space. In the first sub-Section we identify $n \& k$ regions of interest for imaging improvement. In the second sub-Section we dive deeper into the simulation results of foundry N5 building blocks using different mask absorber $n \& k$. This leads us to the identification of materials with predicted imaging gain.

\subsection{Material space vs. reference TaBN mask absorber}

When we look for alternative mask absorber materials from an imaging perspective, we represent the material options by their EUV optical properties, $n \& k$, as these are - together with the absorber thickness - accountable for the imaging response in rigorous lithographic simulators. Figure 1 plots the EUV $n \& k$ space with the measured values for TaBN [17].

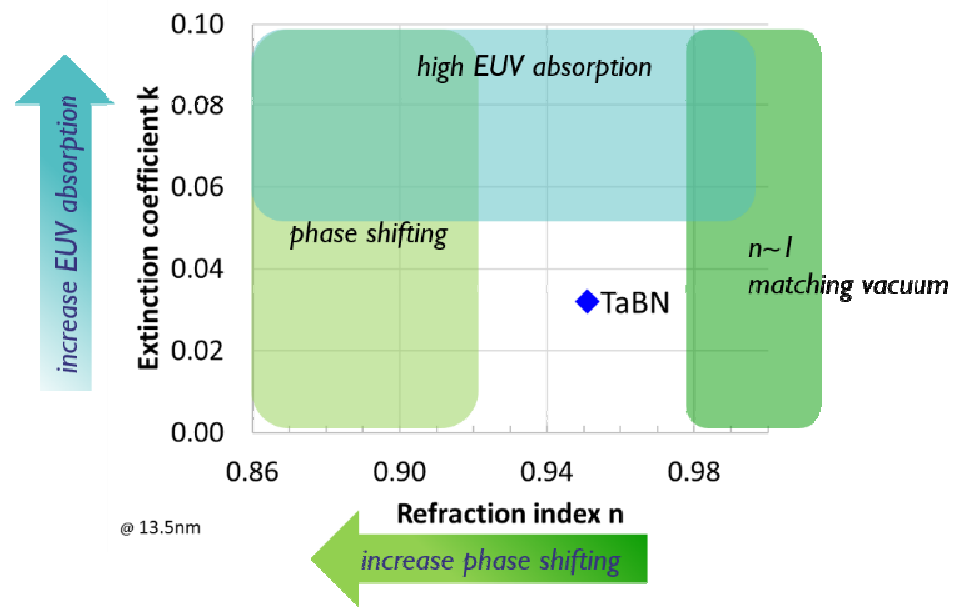

Figure 1 EUV $n \& k$ space with TaBN as the diamond and three possible $n \& k$ regions for novel absorber materials: high EUV absorption, phase matching to vacuum and intentional phase shifting.

From TaBN as starting point we can improve to materials with higher EUV extinction coefficient, which allows for absorber thickness reduction and consequently smaller shadowing effect due to its increased EUV absorption. Tuning the refraction index of the material allows in one direction to reduce the phase deformation, when $n$ is matched to vacuum, and in the other direction to increase the contrast, when enhancing the phase shift character of the material.

\subsection{Imaging performance of $\boldsymbol{n} \boldsymbol{k} \boldsymbol{k}$ regions vs. reference TaBN mask absorber}

We studied the imaging performance of various mask absorbers in the three regions as indicated in Figure 1 for foundry equivalent N5 dedicated building blocks using the rigorous mask 3D simulator S-Litho EUV (Synopsys) [18] including our calibrated and experimentally validated mask Mo/Si multilayer mirror model presented in [19]. Table 1 depicts the illumination settings at NA0.33 and selected feature types and ranges. The $60 \mathrm{~nm}$ reference TaBN mask absorber is compared to candidate absorber materials at $32 \mathrm{~nm}$ thickness with $n \& k$ combinations as represented in Table 1 (c). 
Table 1 (a) Illumination settings at $0.33 \mathrm{NA}$, (b) feature types through pitch, and (c) mask absorber $n \& k$ used in the rigorous imaging simulations.

(a)

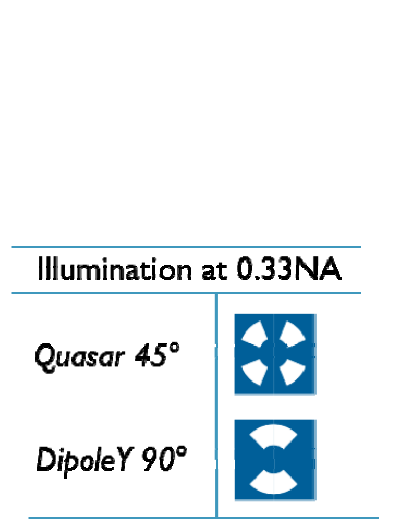

(b)

\begin{tabular}{c|c}
\hline Feature & Pitch range \\
\hline$\overline{2}$ & \\
\hline trench & $32-100 \mathrm{~nm}$ \\
\hline & \\
\hline contacts & $40-100 \mathrm{~nm}$ \\
\hline $\mathbf{2 B a r}$ & $32-100 \mathrm{~nm}$ \\
\hline
\end{tabular}

(c)



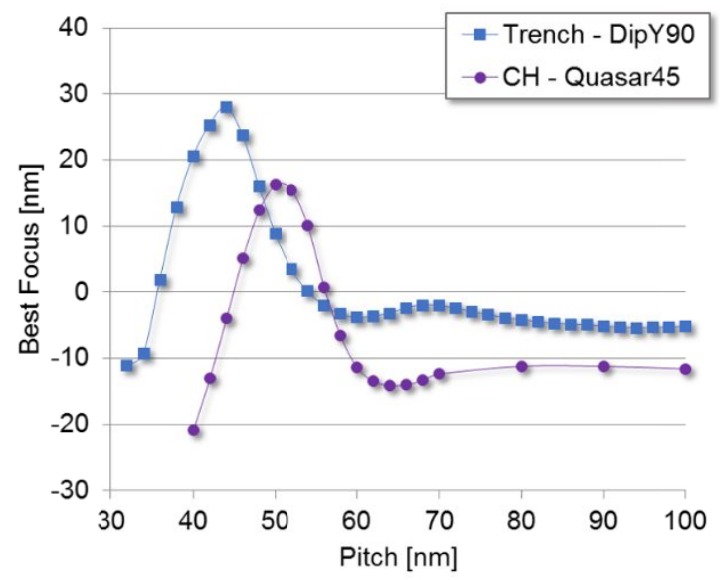


Figure 2 Mask 3D sensitive imaging metrics using the $60 \mathrm{~nm}$ TaBN reference mask for features and illumination at NA0.33 as indicated by the legend in each plot. Left-top: best focus variation through pitch; Right-top: Telecentricity error; Left-bottom: NILS; Right-bottom: Two bar CD asymmetry through focus. 
Quasar45 - Trench - pitch32nm NILS

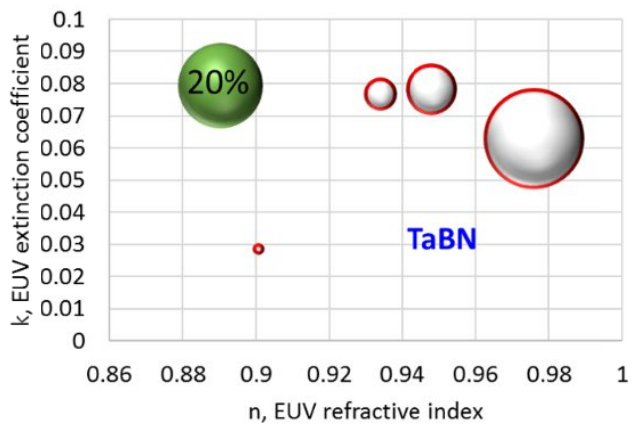

Quasar45 - Trench - Best Focus range

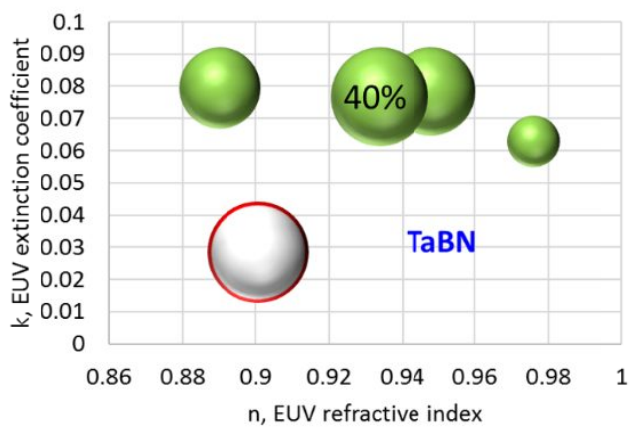

DipoleY90 - Trench - max. telecentr. error

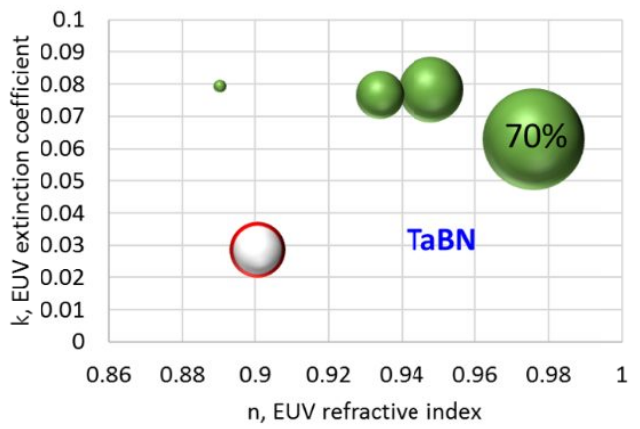

DipoleY90 - pitch32nm 2Bar asymmetry

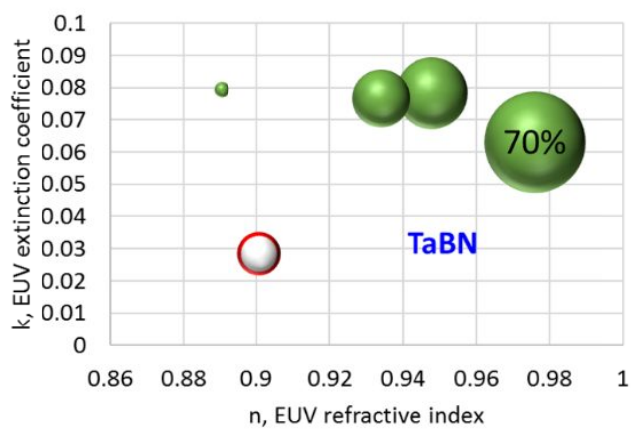

Qs45 - CH - pitch40nm NILS



Qs45 - $\mathrm{CH}$ - Best Focus range



Qs45 - $\mathrm{CH}$ - max. telecentricity error

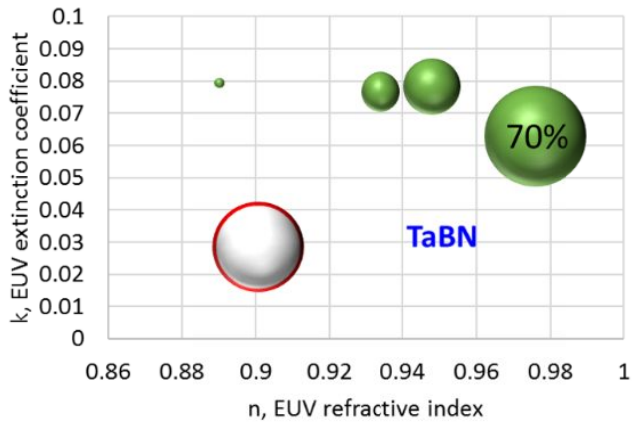

Figure $3 n \& k$ plots with the change in the imaging metric of the $32 \mathrm{~nm} n \& k$ absorber mask compared to the reference $60 \mathrm{~nm}$ TaBN absorber mask. The imaging metric, feature type and illumination at NA0.33 as indicated above the plots. 
The imaging metrics sensitive to mask 3D effects evaluated through pitch for the three feature types and two illumination settings are the best focus variation, the NILS, the telecentricity error (i.e., pattern shift through focus), and the two bar $\mathrm{CD}$ asymmetry through focus. Figure 2 shows the imaging behavior using the $60 \mathrm{~nm}$ TaBN reference mask. The range of best focus through pitch amounts to $40 \mathrm{~nm}$, while the maximum telecentricity error reaches $-13 \mathrm{mrad}$ and two bar CD asymmetry through focus at the smallest pitch of $32 \mathrm{~nm}$ extents to $48 \mathrm{mrad}$. Improving the imaging performance compared to the reference mask implies that the NILS needs to increase, while the best focus range, the maximum telecentricity error and two bar CD asymmetry through focus need to decrease. The relative change of each of these metrics by changing the absorber material with respect to the reference is plotted in $n \& k$ plots in Figure 3 . The size of the circle symbols is relative to the reference TaBN. The green color implies an improvement of the metric, while a red circle refers to a deterioration of the metric compared to the behavior of the reference mask. Also indicated in each plot is the value of the largest relative improvement. Combining all the imaging results allows us to identify regions in the $n \& k$ space with expected imaging improvement compared to the reference mask. The resulting plot is shown in Figure 4. A region of increased NILS can be observed for materials with a refraction index $n$ smaller than that of TaBN. This can be attributed to a destructive interference of (increasingly) phase shifted light in the absorber area and light in the vacuum region of the mask. Moreover, the decreased refraction index contrast between the absorber material and the vacuum causes a more pronounced waveguide effect for the light in the vacuum spaces and a further increase of the NILS. This finding supports earlier work [2] where a co-optimization of mask material and illumination through multiobjective optimization led to the improved imaging of an attenuated phase shifting mask compared to the reference Tabased mask. A region of smaller best focus range through pitch can be found at higher extinction coefficient $k$ than TaBN due to stronger EUV absorption over a smaller absorber thickness minimizing the phase impact. Lower telecentricity errors and two bar CD asymmetry through focus can be found at a refraction index $n$ close to 1 due to the phase matching of the absorber material to the surrounding vacuum. The weight given to each optimization metric determines the optimal $n \& k$ region, although increasing the extinction coefficient $k$ compared to TaBN points to a general improvement.

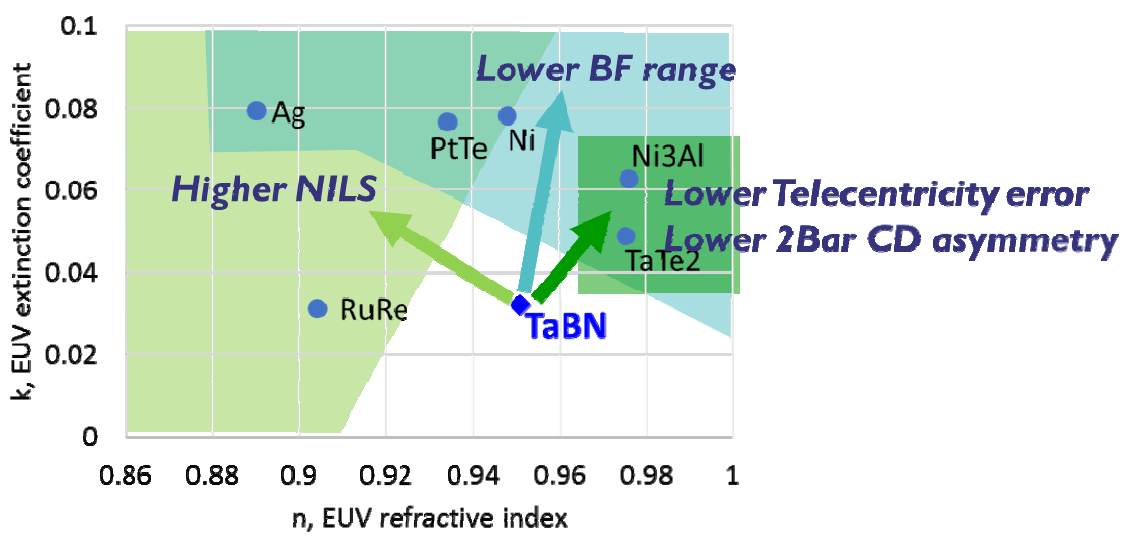

Figure $4 n \& k$ plot indicating three regions with expected improved imaging performance compared to the reference TaBN.

Within each of these regions we engineered and characterized potential EUV mask absorber materials. Ag, Ni and PtTe reside in the region of high extinction coefficient. $\mathrm{Ni}_{3} \mathrm{Al}$ and $\mathrm{TaTe}_{2}$ exhibit a refraction index approaching one, while RuRe is positioned in the attenuated phase shifting region. Figure 4 illustrates the $n \& k$ of the materials studied in more detail in the next Section.

\section{EUV MASK ABSORBER REQUIREMENTS}

Mask absorber change is a complex and difficult process in the mask making technology, since the candidate material needs to fulfil a diversity of requirements to guarantee success throughout the complete EUV mask life cycle. Blank manufacturing, mask fabrication and qualification, and mask use pose various requirements on the candidate absorber. A more extensive list can be found in [20]. Table 2 lists the requirements we investigated in more details in the following sub-Sections. 
Table 2 Investigated mask absorber requirements with their impact on EUV lithography and the testing methodology.

\begin{tabular}{|l|l|l|l|}
\hline \hline Characterization & Evaluation & impacts & Testing methodology \\
\hline \hline \multirow{3}{*}{ Film morphology } & $\begin{array}{l}\text { Crystallinity } \\
\text { Surface composition } \\
\text { Surface roughness }\end{array}$ & $\begin{array}{l}\text { Line edge roughness } \\
\text { Film stability } \\
\text { Out-of-band }\end{array}$ & $\begin{array}{l}\text { TEM, IS-XRD } \\
\text { XPS, TEM } \\
\text { AFM }\end{array}$ \\
\hline Optical constants & EUV n\&k & Imaging & EUV reflectometry \\
\hline \multirow{2}{*}{ Mask durability } & $\begin{array}{l}\text { EUV \& H* interaction } \\
\text { Wet clean }\end{array}$ & $\begin{array}{l}\text { Usage in scanner } \\
\text { Mask cleaning }\end{array}$ & $\begin{array}{l}\text { H* cleaner, high power } \\
\text { EUV } \\
\text { ICP-MS }\end{array}$ \\
\hline Mask patterning & Traditional or disruptive & Pattern profile & RIE or ASD \\
\hline \hline
\end{tabular}

\subsection{Film morphology}

Single metal films deposited by physical vapor deposition (PVD) suffer from full-layer crystallization and islandformation $[1,20]$. In the absorber films, studied in this work, the poly-crystallinity is reduced by multilayering or alloying.

Ag-based layered structures have been developed as potential alternative binary absorbers. Ag exhibits one of the largest extinction coefficients at $13.5 \mathrm{~nm}$, and as such is of interest for absorber development. The problem of full layer crystallization and islanding/roughness development during sputter deposited $\mathrm{Ag}$ growth was addressed by using an adhesive layer, such as Te or $\mathrm{Cu}$. Further build-up of crystallinity and roughness is repressed by using a multi-layered structure, with individual layers of a few nanometers. Structural characterization of the $\mathrm{Ag} / \mathrm{Te}$ system with X-ray diffractometry (XRD) (see Figure 5(a)) showed moderately reduced crystallite sizes, compared to pure Ag, coupled to a surface roughness with peak-to-valley in the few-nanometer range. To further reduce the film crystallinity and roughness, $\mathrm{Ag} / \mathrm{Cu}$ multilayers have been developed that exhibit nanometer size crystals, have a rms roughness of 0.25 $\mathrm{nm}$, and are less prone to oxidation than $\mathrm{Te}$. Changing the fraction of $\mathrm{Ag}$ and $\mathrm{Cu}$ only shifts the balance from nanometer size $\mathrm{Ag}$ to nanometer size $\mathrm{Cu}$ crystallites, both far away from full layer crystallization, as presented in Figure 5(b). The ratio of $\mathrm{Ag}$ to $\mathrm{Cu}$ can be further explored in order to tailor the optical response of the multilayer absorber, and as such $\mathrm{Ag} / \mathrm{Cu}$ multilayers provide from this perspective a promising candidate for application as binary absorber material.
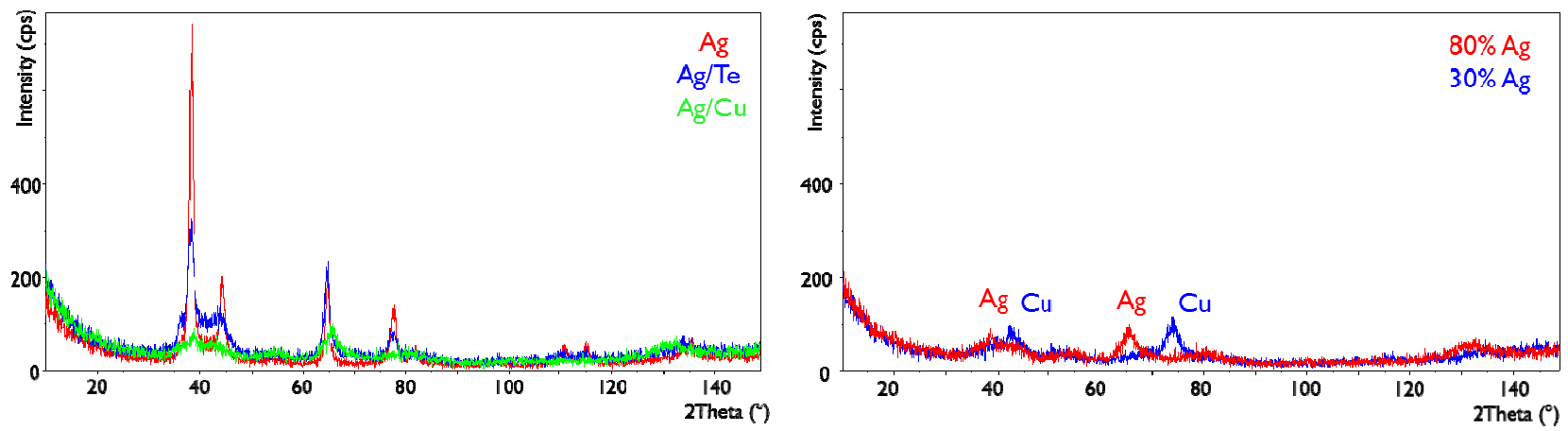

Figure 5 (a) XRD spectra for a single $\mathrm{Ag}$ layer, $\mathrm{Ag} / \mathrm{Te}$ and $\mathrm{Ag} / \mathrm{Cu}$ multilayers. $\mathrm{Ag} / \mathrm{Cu}$ multilayers show very low crystallinity. (b) XRD comparison of crystallinity in low and high $\mathrm{Cu}$ containing $\mathrm{Ag} / \mathrm{Cu}$ multilayers.

Alloying the suitable optical property of individual elements in a stable composition is another approach to reduce the film poly-crystallinity. In our earlier work we already reported on the development, characterization and imaging improvement of Ni-Al alloys [20]. To ensure high EUV absorption, we engineered metal telluride alloys with at least $50 \% \mathrm{Te}$ due to its high EUV absorption. Our development includes Ru-alloys intended for EUV attenuated phase shifting materials [2].

To increase the chemical stability of Te, we have investigated the noble metal telluride Pt-Te. The transmission electron microscope (TEM) image in Figure 6(a) shows the quasi-amorphous morphology of as-deposited PtTe, which is confirmed by in-situ XRD (IS-XRD) measurements, where it is shown that recrystallization occurs at $210^{\circ} \mathrm{C}$. 
We investigated the feasibility of Ta-telluride. The IS-XRD measurements and TEM image in Figure 6(b) prove the quasi-amorphous morphology of as-deposited Ta-Te alloy. A change in background intensity can be detected above $350^{\circ} \mathrm{C}$, which will be discussed in more detail in Section 3.3.1. Furthermore, the presence of oxygen is detected through the full absorber layer.

As-deposited Ru-alloys exhibit a high degree of poly-crystallinity, visible in the TEM image of $\mathrm{Ru}_{3} \mathrm{Re}$ in Figure $6(\mathrm{c})$. This crystalline phase remains the same towards $500^{\circ} \mathrm{C}$, based on the IS-XRD measurements. Doping with smaller elements, such as $\mathrm{N}$ and $\mathrm{P}$, might reduce the crystallinity, by breaking the Ru crystal lattice through their size difference [21-22].
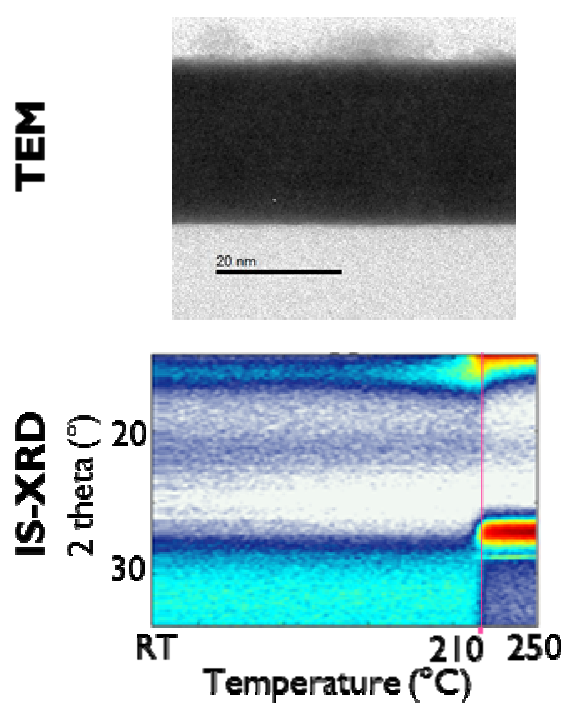

(a)



(b)

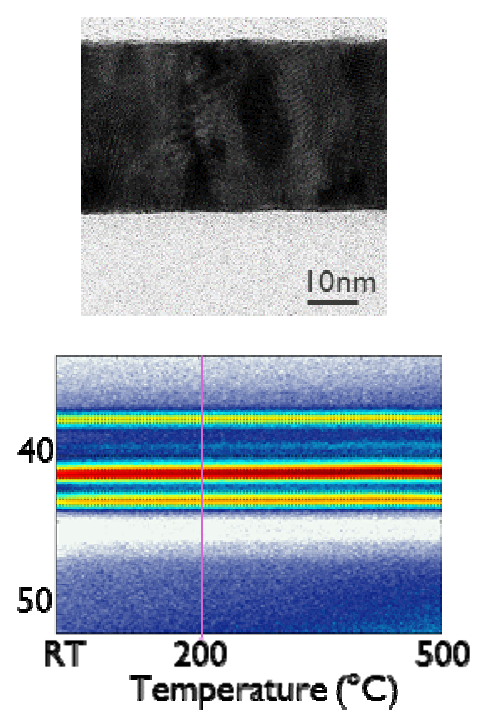

(c)

Figure 6 Top: TEM image of as-deposited (a) PtTe, (b) $\mathrm{TaTe}_{2}$, and (c) $\mathrm{Ru}_{3} \mathrm{Re}$ film. Bottom: corresponding IS-XRD plot.

\subsection{EUV optical properties}

The EUV optical properties of the mask absorber material and its thickness determine its ability to mitigate mask induced imaging effects. EUV reflectance measurements through incidence angle and wavelength (from $10 \mathrm{~nm}$ to 16 $\mathrm{nm}$ ) are conducted in the lubrication free EUV reflectometer of PTB at the soft X-ray radiometry beamline at the BESSY II storage ring [17]. The obtained surface plot, presented for three alloys in Figure 7, is fitted to provide the $n \& k$ values in the wavelength range, as well as the thickness of the individual layers in the film. The accurate $n \& k$ measurement of the engineered alloys is essential to correctly predict the imaging impact of these potential mask absorber films in rigorous lithographic simulation (cf. Section 2.2).

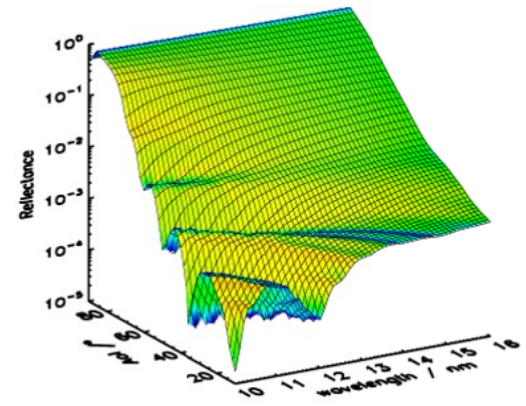

(a)



(b)

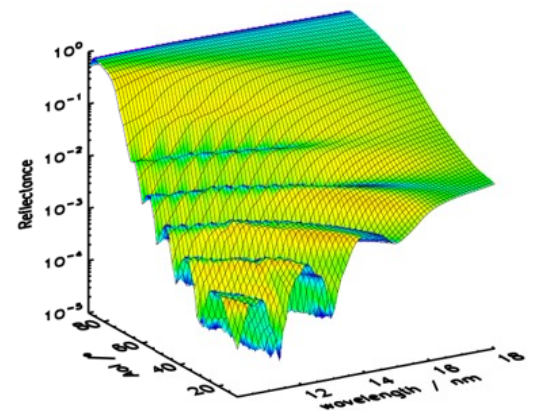

(c)

Figure 7 EUV reflectance (in $\log 10$ scale) as function of wavelength and incidence angle of a $\sim 30 \mathrm{~nm}$ thick (a) PtTe film, (b) $\mathrm{Ni}_{3} \mathrm{Al}$ film and (c) $\mathrm{Ru}_{3} \mathrm{Re}$ film on Si substrate. 


\subsection{Absorber durability}

During normal mask usage in the fab the mask absorber must stay unaltered in scanner conditions under normal operation. Additionally, the mask needs to withstand several wet conditions, when cleaned for removing surface particles. We have a testing methodology in place to assess the thermal stability as well as the stability under hydrogen environment and in solutions of the candidate material films. This stability quantification of the engineered absorber films allows us to make a proper absorber selection.

\subsubsection{Thermal stability}

The temperature of the mask in the scanner or in storage will vary, but it may not impact the material morphology.

We quantified the lifetime of the candidate absorber films by calculating the recrystallization activation energy through a Kissinger analysis [23]. For the PtTe film we extrapolated the lifetime until recrystallization at different temperatures as presented in Figure 8(a). At a constant temperature of $80^{\circ} \mathrm{C}$ the PtTe film will recrystallize after 450 years, which is beyond the typical mask lifetime in a fab.

Under thermal loading the alloy might suffer from material loss. For the $\mathrm{TaTe}_{2}$ and $\mathrm{PtTe}$ films the Te loss was measured at different temperatures, as shown in Figure 8 (b-c). Te becomes volatile just above $400^{\circ} \mathrm{C}$ and therefore in both tellurides a significant Te loss is detected at $500^{\circ} \mathrm{C}$. At a temperature of $250^{\circ} \mathrm{C}$ the noble metal telluride PtTe seems to stabilize the Te better than the Ta-telluride, where some Te loss is noticed.



(a)

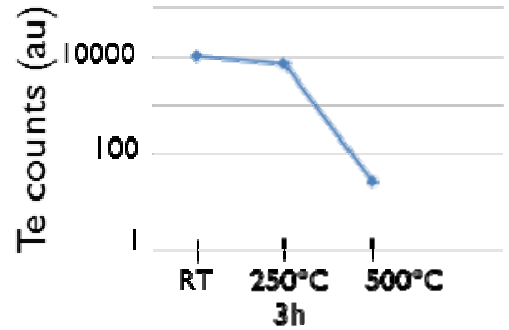

(b)

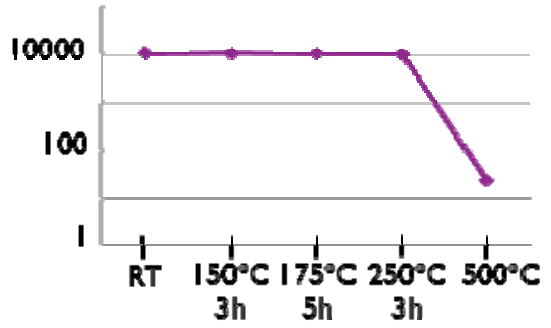

(c)

Figure 8 (a) Lifetime until recrystallization of PtTe film vs. temperature. Te loss measured at different thermal loadings for (b) $\mathrm{TaTe}_{2}$ and (c) PtTe film.

From the thermal stability tests on the engineered absorber films we learned that $\mathrm{PtTe}$ and $\mathrm{TaTe}_{2}$ will stay unaltered during typical mask thermal conditions (i.e., below $150^{\circ} \mathrm{C}$ ).

\subsubsection{Wet conditions}

The candidate mask absorber must stay stable during typical mask cleaning. We selected two solutions currently used in mask cleaning: (1) de-ionized water (DIW) at $\mathrm{pH} 5.7,(2) 1 \% \mathrm{NH}_{4} \mathrm{OH}$ at $\mathrm{pH} 11.4$. First the samples are fully submersed in a beaker with the solutions for $\sim 24$ hours. After visual inspection the films are measured by XRR to determine film thickness loss, roughening or density change. The stability of the alloys in wet conditions depends on the reactivity of each surface element. Aluminum metal reacts under both acidic and alkalic conditions, which impacts the reaction of $\mathrm{Ni}_{3} \mathrm{Al}$ in both tested aqueous solutions. $\mathrm{TaTe}_{2}$ and PtTe are more stable in the solutions due to the stability of Ta and Pt respectively.

Next, the PtTe film is characterized by the highly sensitive technique of inductively coupled plasma mass spectrometry (ICP-MS) to determine the rate of material loss in each aqueous solution. Figure 9(a) shows the measured Te dissolved in both aqueous solutions after three different time durations. 


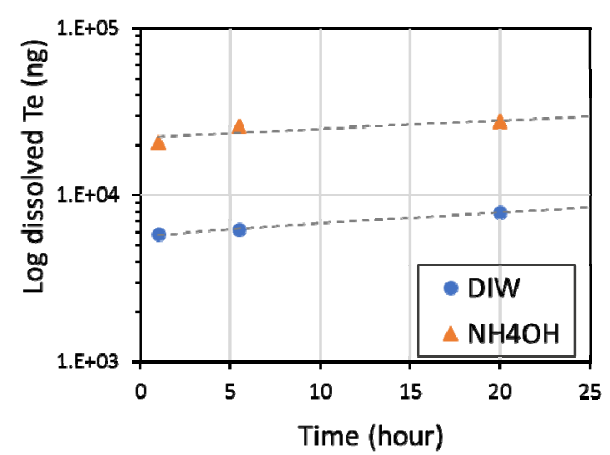

(a)

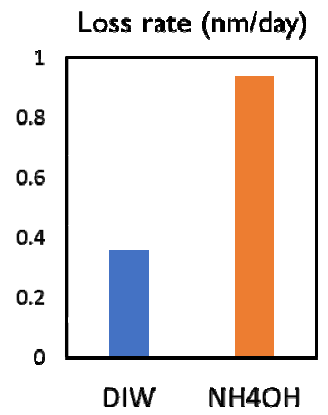

(b)

Figure 9 (a) Dissolved Te measured by ICP-MS in two different aqueous solutions after three different time durations. (b) Calculated Te loss rate per full day in two different aqueous solutions.

From the slope through time we calculate the Te dissolution after a full day of submersion to be less the $0.4 \mathrm{~nm}$ in DIW and less than $1 \mathrm{~nm}$ in $1 \% \mathrm{NH}_{4} \mathrm{OH}$. The quantification of absorber material loss after multiple mask cleans allows us to perform an imaging sensitivity study in a next stage.

\subsubsection{Scanner conditions}

In the scanner the mask, and thus the mask absorber material, is exposed to high EUV power in $\mathrm{H}_{2}$ environment. Under these operation conditions hydrogen radicals $\left(\mathrm{H}^{*}\right)$ can be formed and these $\mathrm{H}^{*}$ can react with the absorber material. However, the absorber material is not allowed to form volatile contaminants or to change its film morphology. Therefore, we conducted a first assessment by exposing our alloy films to a strong $\mathrm{H}^{*}$ environment in imec's EUV Tech hydrogen cleaner [20]. The elemental content of the films before and after exposure was determined by Rutherford backscattering spectrometry (RBS) and the relative changes are plotted in Figure 10. The $\mathrm{Ni}_{3} \mathrm{Al}$ and $\mathrm{Ru}_{3} \mathrm{Re}$ films remain intact after $\sim 24$ hours $\mathrm{H}^{*}$ exposure, while the tellurides lose Te content due to reaction to $\mathrm{H}^{*}$. Again, the noble metal telluride PtTe seems to bind the Te stronger in the bulk than the Ta-telluride.

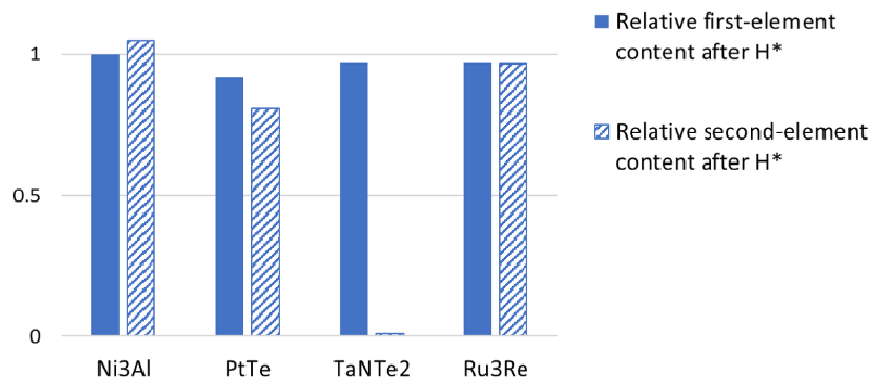

Figure 10 Relative elemental content after more than 24 hours $\mathrm{H}^{*}$ exposure as determined by RBS.

The individual $\mathrm{H}^{*}$ test with the novel absorber films provides a rough estimate of possible interactions. However, the test conditions to pure $\mathrm{H}^{*}$ environment are too aggressive compared to the actual scanner conditions. A dedicated test setup is required to effectively assess the impact of combined high EUV power and $\mathrm{H}_{2}$ atmosphere. We performed a feasibility test in an irradiation chamber at the beamline of PTB where the PtTe films were exposed to variable conditions of combined EUV power density and $\mathrm{H}_{2}$ gas pressure [24]. Our initial results in Figure 11 are obtained by characterizing the films after EUV $+\mathrm{H}_{2}$ exposure by X-ray photoelectron spectroscopy (XPS). 


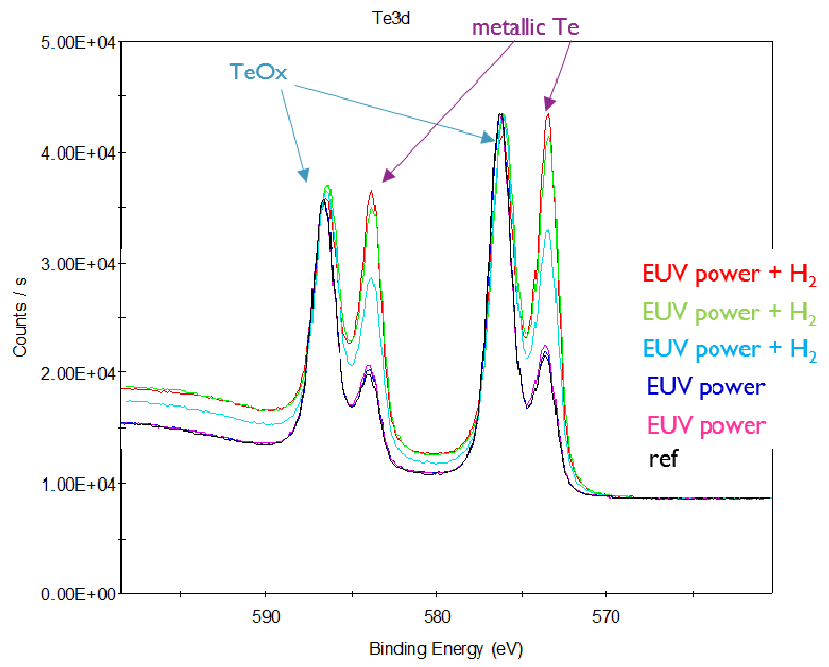

Figure 11 Normalized XPS spectra of Te3d peaks from the reference and five PtTe films exposed to different EUV+ $\mathrm{H}_{2}$ conditions.

Two groups of results can be observed: the reference PtTe film and the PtTe films only exposed to EUV show strong oxidized Te peaks at the PtTe surface, while a combination of EUV and $\mathrm{H}_{2}$ gas seems to reduce the Te-oxide on the PtTe surface to metallic Te, resulting in equal Te-oxide and metallic Te peaks.

These initial results indicate the sensitivity of the materials to the combined exposure of EUV power and $\mathrm{H}_{2}$ environment, but the samples need additional characterization to quantify its impact. Further optimization of the test conditions and matching to actual scanner conditions is ongoing.

\subsection{Absorber patterning}

To emphasis the importance of absorber patterning we assessed experimentally the feasibility of two patterning approaches. Subtractive patterning is the traditional way of patterning the mask absorber, while additive patterning can be considered a disruptive manner for the mask making technology.

\subsubsection{Subtractive patterning}

Mask absorber patterning is typically achieved with reactive ion etching (RIE), which is a chemically assisted physical etching process. By forming volatile compounds, the etching is material selective. The physical etching by ion beam sputtering can pattern through thin metal layers anisotropically, but it is not extremely material selective. From earlier work [1] we experienced the challenges of metal patterning through RIE etching. Now we focus on the chemical component of this etch technique by using halogen-based plasma only, without a carrier gas to reduce the physical etch, and to demonstrate volatile formation with the elemental components of the absorber alloy.

In a first etching test a $1 \mu \mathrm{m}$ thick patterned resist film is used as mask for the chemical etching of the $\sim 30 \mathrm{~nm}$ thick material film in an RIE tool. The cross-section SEM images in Figure 12 illustrate the outcome for the pure Te, the PtTe and the $\mathrm{TaTe}_{2}$ film.

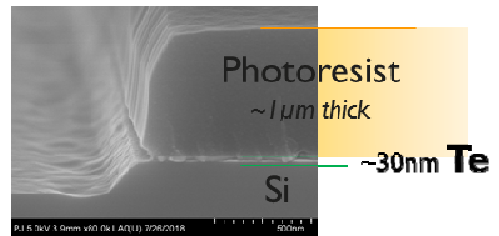

(a)



(b)



(c)

Figure 12 Cross-section SEM images after chemical etch in halogen-based plasma of $\sim 30 \mathrm{~nm}$ thick (a) Te, (b) $\mathrm{TaTe}_{2}$, and (c) PtTe film on Si substrate and with a $1 \mu \mathrm{m}$ thick resist pattern on top. 
The pure Te film is isotropically etched in the plasma and is therefore likely etchable by RIE. The Ta-telluride is also removed in the chemical etch process. This is a strong indication that alloys with Te and other chemically etchable materials are patternable in a subtractive way. However, the Pt-telluride sample suffers from fences on the resist sidewalls and from residues in the removed trenches, which is typical for metal etching with limited volatile byproducts, as we also observed for Ni etch [1].

High- $k$ metals, like $\mathrm{Ni}$ and $\mathrm{Pt}$, are difficult to etch selectively with known chemistries. Direct metal etch process development is needed to solve these patterning challenges.

\subsubsection{Additive patterning}

Because subtractive metal patterning poses challenges on selectivity and chemical volatility of metal elements, we took a disruptive approach of metal-on-metal area selective deposition (ASD) for mask patterning purpose [25].

Since our simulation study converges to high- $k$ metals as candidate mask absorber materials, and the multilayer mirror of an EUV mask is capped with Ru, which is a metal, we can grow in a controllable way metal selectively on metal by using a dielectric template through metal electroless deposition [26]. The flow is exemplified in Figure 13.

We start with sacrificial patterning in a dielectric layer (e.g., $\mathrm{SiO}_{2}$ ). The quality of the template pattern will determine the sidewall and edge of the final metal features. The dielectric patterning enables further $\mathrm{CD} /$ pitch scaling with good sidewall definition. In a next step the ASD is performed where the metal (i.e., the mask absorber material) grows in a controllable way on the open Ru metal areas in between the template. In our example cobalt ASD is performed and in Figure 13 (b) the trenches in the template are filled with cobalt without Co deposition on top of the template. Furthermore, the $\mathrm{Ru}$ capping layer is not damaged by additive patterning, contrary to etching. In the last step the template is removed to result in the final Co metal absorber pattern. Characterization of the Co ASD in this example shows a fully amorphous cobalt pattern, as crystallization is confined by the dielectric template. This is an additional advantage of the additive patterning technique, while Co PVD is poly-crystalline. Additive patterning has potential to create amorphous metal patterns on the Ru cap of the multilayer mirror. Further optimization is required to demonstrate the compatibility with mask size, e.g., in terms of thickness uniformity and defectivity.

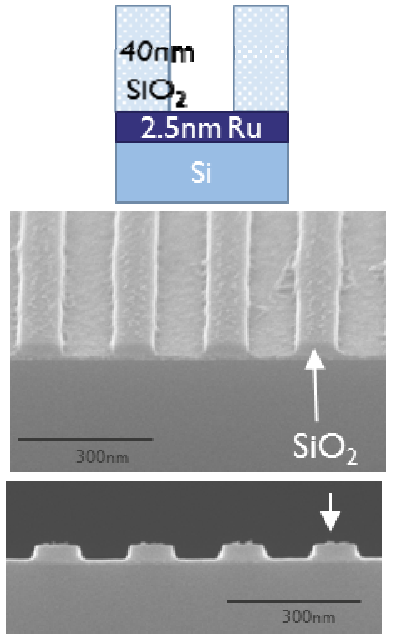

(a)



(b)

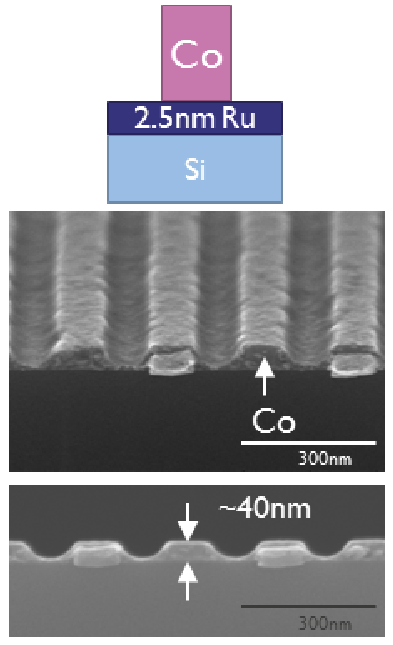

(c)

Figure 13 Simplified flow for metal-on-metal ASD on 200nm pitch equal lines/spaces. (a) Template patterning; (b) cobalt ASD; (c) Co pattern after template removal.

\section{CONCLUSION}

In this paper we proposed novel EUV mask absorbers based on a combined assessment of their imaging performance and experimental behavior in EUV lithography conditions. We identified EUV $n \& k$ regions for mask absorbers around $32 \mathrm{~nm}$ thickness with improved mask induced imaging effects compared to the current Ta-based mask absorber. For each of these improvement regions, we engineered novel mask absorbers. The PtTe alloy and Ag-based multilayer have a high EUV absorption and therefore will suffer less from best focus variations. NiAl and TaTe alloys match at EUV 
wavelength their phase close to vacuum resulting in reduced telecentricity errors and two bar CD asymmetry through focus. The RuRe alloy is put forward as potential attenuated phase shifting material for EUV masks expected to increase NILS at reduced dose-to-size with the proper mask-illumination optimization.

We presented the experimental testing methodology geared to address several essential mask absorber requirements to allow for a material down-selection. Our testing flow assesses the durability of the film morphology under different thermal, EUV, hydrogen, and cleaning conditions, typical for mask environment. We found that the most interesting materials from lithographic perspective pose challenges on traditional mask etching technology. Adapting towards etchable materials creates a trade-off in material durability and subtractive patterning. Therefore, we introduced the concept of additive patterning for mask absorbers.

Our next goal is to find solutions - through collaborative efforts - for the absorber patterning challenges of the proposed novel mask absorber materials.

\section{ACKNOWLEDGEMENTS}

This project has received funding from the Electronic Component Systems for European Leadership Undertaking under grant agreement number 662338. This Joint Undertaking receives support from the European Union's Horizon 2020 research and innovation programme and Netherlands, France, Belgium, Germany, Czech Republic, Austria, Hungary, Israel.

The authors are grateful to Dr. I. Pollentier and Dr. J. Rip for their assistance in the durability testing. Dr. L. Souriau is acknowledged for his etch support and expertise. For metrology support we thank N. Vandenbroeck, P. Jaenen, Dr. T. Conard, Dr. I. Hoflijk, and Dr. J. Meersschaut (imec). Area selective deposition of Co was made possible thanks to K. Vandersmissen and Dr. BT Chan (imec). We appreciate the support of Dr. G. McIntyre, Dr. K. Ronse, Dr. S. Van Elshocht (imec), and Prof. Dr. M. Heyns (KU Leuven).

\section{REFERENCES}

1 Philipsen, V., Luong, K.V., Souriau, L., Erdmann, A., Evanschitzky, P., van de Kruijs, R.W. E., Edrisi, A., Scholze, F., Laubis, C., Irmscher, M., Naasz, S., Reuter, C., Hendrickx, E., "Reducing extreme ultraviolet mask three-dimensional effects by alternative metal absorbers," Journal of Micro/Nanolithography, MEMS, and MOEMS 16 (4), 041002 (2017).

2 Erdmann, A., Evanschitzky, P., Mesilhy, H., Philipsen, V., Hendrickx, E., Bauer, M., “Attenuated phase shift mask for extreme ultraviolet: can they mitigate three-dimensional mask effects?," Journal of Micro/Nanolithography, MEMS, and MOEMS 18 (1), 011005 (2018).

3 Erdmann, A., Fühner, T., Evanschitzky, P., Neumann, J.T., Ruoff, J., Gräupner, P., "Modeling studies on alternative EUV mask concepts for higher NA," Proc. SPIE 8679, 86791Q (2013).

4 Erdmann, A., Xu, D., Evanschitzky, P., Philipsen, V., Luong, V., Hendrickx, E., "Characterization and Mitigation of 3D Mask Effects in Extreme Ultraviolet Lithography," Advanced Optical Technologies 6, 187 (2017).

5 Finders, J., de Winter, L., Last, T., "Mitigation of mask three-dimensional induced phase effects by absorber optimization in ArFi and extreme ultraviolet lithography," Journal of Micro/Nanolithography, MEMS, and MOEMS 15 (2), 021408 (2016).

6 Yan, P.Y., "The impact of EUVL mask buffer and absorber material properties on mask quality and performance," Proc. SPIE 4688, 150 (2002).

7 Burkhardt, M., "Investigation of alternate mask absorbers in EUV lithography," Proc. SPIE 10143, 1014312 (2017).

8 Wood, O., Raghunathan, S., Mangat, P., Philipsen, V., Luong, V., Kearney, P., Verduijn, E., Kumar, A., Patil, S., Laubis, C., Soltwisch, V., Scholze, F., "Alternative materials for high numerical aperture extreme ultraviolet lithography mask stacks,” Proc. SPIE 9422, 94220I (2015).

9 Erdmann, A., Evanschitzky, P., Neumann, J.T., Gräupner, P., "Mask-induced best-focus-shifts in DUV and EUV lithography," Journal of Micro/Nanolithography, MEMS, and MOEMS 15 (2), 021205 (2016). 
10 Davydova, N., de Kruif, R., Rolff, H., Connolly, B., van Setten, E., Lammers, A., Oorschot, D., Fukugami, N., Kodera, Y., "Experimental approach to EUV imaging enhancement by mask absorber height optimization," Proc. SPIE 8886,888622 (2013).

11 Last, T., L. de Winter, L., Finders, J., "Imaging enhancement by reduction of mask topography induced phase aberrations for horizontal 1D spaces under D90Y illumination," Proc. SPIE 9635, 96350K (2015).

12 Philipsen, V., Hendrickx, E., Verduijn, E., Raghunathan, S., Wood, O., Soltwisch, V., Scholze, F., Davydova, N., and Mangat, P., "Imaging impact of multilayer tuning in EUV masks, experimental validation," Proc. SPIE 9235, 923518 (2014).

13 Wood, O., Wong, K., Parks, V., Kearney, P., Meyer-Ilse, J., Luong, V., Philipsen, V., Faheem, M., Liang, Y., Kumar, A., Chen, E., Bennett, C., Fu, B., Gribelyuk, M., Zhao, W., Mangat, P., van der Heide, P., "Improved Ru/Si multilayer reflective coatings for advanced extreme ultraviolet lithography masks," Proc. SPIE 9776, 977619 (2016).

14 Takai, K., Motokawa, T., Koji, M., Kamo, T., Hayashi, N., "Patterning of EUVL binary etched multilayer mask," Proc. SPIE 8880, 88802M (2013).

15 Van Look, L., Philipsen, V., Hendrickx, E., Vandenberghe, G., Davydova, N., Wittebrood, F., de Kruif, R., van Oosten, A., Miyazaki, J., Fliervoet, T., van Schoot, J., Neumann, J.T., "Alternative EUV mask technology to compensate for mask 3D effects,” Proc. SPIE 9658, 96580I (2015).

16 imec Technology Forum on advanced EUV mask absorbers at SPIE AL 2018 and PUV 2018.

17 Scholze, F., Laubis, C., Luong, K.V., Philipsen, V., "Update on optical material properties for alternative EUV mask absorber materials," Proc. SPIE 10446, 1044609 (2017).

18 http://www.synopsys.com/silicon/mask-synthesis/Sentaurus-Lithography.html

19 Philipsen, V., Hendrickx, E., Jonckheere, R., Davydova, N., Fliervoet, T., and Neumann, J.T., "Actinic characterization and modeling of the EUV mask stack," Proc. SPIE 8886, 19 (2013).

20 Luong, V., Philipsen, V., Hendrickx, E., Opsomer, K., Detavernier, C., Laubis, C., Scholze, F., Heyns, M., "Ni-Al alloys as alternative EUV mask absorber," Appl. Sci. (8), 521 (2018).

21 Shin, J., Waheed, A., Agapiou, K., Winkenwerder, W.A., Kim, H.W., Jones, R.A., Hwang, G.S., Ekerdt, J.G., "Growth of ultrathin films of amorphous ruthenium-phosphorus alloys using a single source CVD precursor," J. Am. Chem. Soc. 128 (51), 16510 (2006).

22 Wu, C.Y., Lee, W.H., Chang, S.C., Cheng, Y.L., Wang, Y.L., "Effect of annealing on the microstructure and electrical property of RuN thin films," J. Electrochem. Soc. 158 (3), H338 (2011).

23 Devulder, W., Opsomer, K., Minjauw, M.M., Meersschaut, J., Jurczak, M., Goux, L., Detavernier, C., "Study of amorphous $\mathrm{Cu}-\mathrm{Te}-\mathrm{Si}$ thin films showing high thermal stability for application as a cation supply layer in conductive bridge random access memory devices," RSC Adv. 6, 32106 (2016). A detailed report on the crystallization kinetics will be covered in a forthcoming paper of K. Opsomer, et al.

24 Klein, R., Scholze, F., Thornagel, R., Tummler, J., Wedowski, M., Jansen, R., Mertens, B., van de Runstraat, A., Ulm, G., "Irradiation of EUV multilayer optics with synchrotron radiation of a different time structure," Proc. SPIE 4782, 292 (2002).

25 Philipsen, V., Luong, K.V., Souriau, L., Altamirano-Sanchez, E., Adelmann, C., Hendrickx, E., Scholze, F., Laubis, C., Kruemberg, J., Reuter, C., "Single element and metal alloy novel EUV mask absorbers for improved imaging," Proc. SPIE 10450 (2017), International Conference on Extreme Ultraviolet Lithography 2017.

26 Kolics, A., "Electroless technology for the upcoming challenges in interconnect metallization," ECS Transactions $60(1), 431(2014)$. 